\title{
Long Reach Robotic Arm
}

Amanda Hoeksema \& Brenda Sanchez Under the mentorship of Kris Anderson AD Robotics Initiative - $\mathrm{CCl}$ 5 August $2020 \quad \begin{aligned} & \text { This manuscript has been authored by Fermi Research Alliance, LLC under Con } \\ & \text { U.S. Department of Energy, Office of Science, Office of High Energy Physics. }\end{aligned}$ 


\section{Long Reach Robotic Arm}

\section{Problem}

- Quadrupole magnets frequent failure

- Water leaks

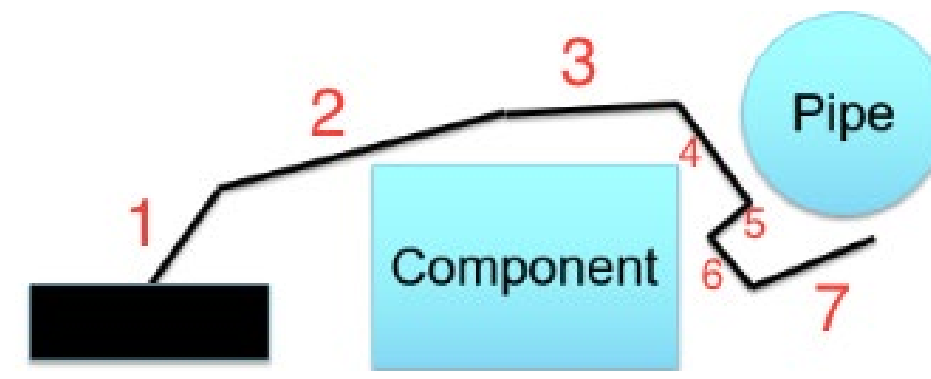

- Critical systems around the magnets that need access

- Tight space of components make it difficult to view

\section{Solution}

- Long robotic arm with many joints to extend and reach around components

- Camera attached at the arm's end 


\section{Long Reach Robotic Arm Design - Split in Two}

- The Arm

- Control certain links of the arm

- Move direction X,Y, \& Z while avoiding components

- "Snake" around obstructions

- Compact design for mobility
- The Base/Counterweight

- Needs to support the extended long arm

- Large enough to provide stability but not consume too much space in small tunnel

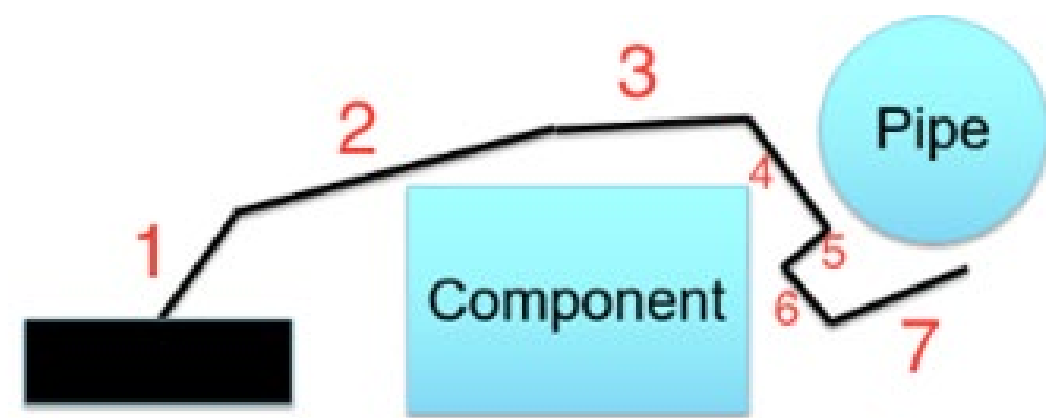





茟 Fermilab 


\section{Coding}

\section{Program For Robot}




\section{Code for Robot}

\section{Enter distance between the center of the base and the wall (in inches):46}

Enter number of arm links (1-6):5

Enter length of Link One (in inches):28

Enter length of Link Two (in inches): 28

Enter length of Link Three (in inches) 24

Enter length of Link Four (in inches):20

Enter length of Link Five (in inches):18

\begin{tabular}{|c|c|}
\hline 1 & \#include 〈stdio.h〉 \\
\hline 2 & \#include 〈stdlib.h〉 \\
\hline 3 &  \\
\hline 4 & \#include 〈math.h〉 \\
\hline
\end{tabular}

Libraries used

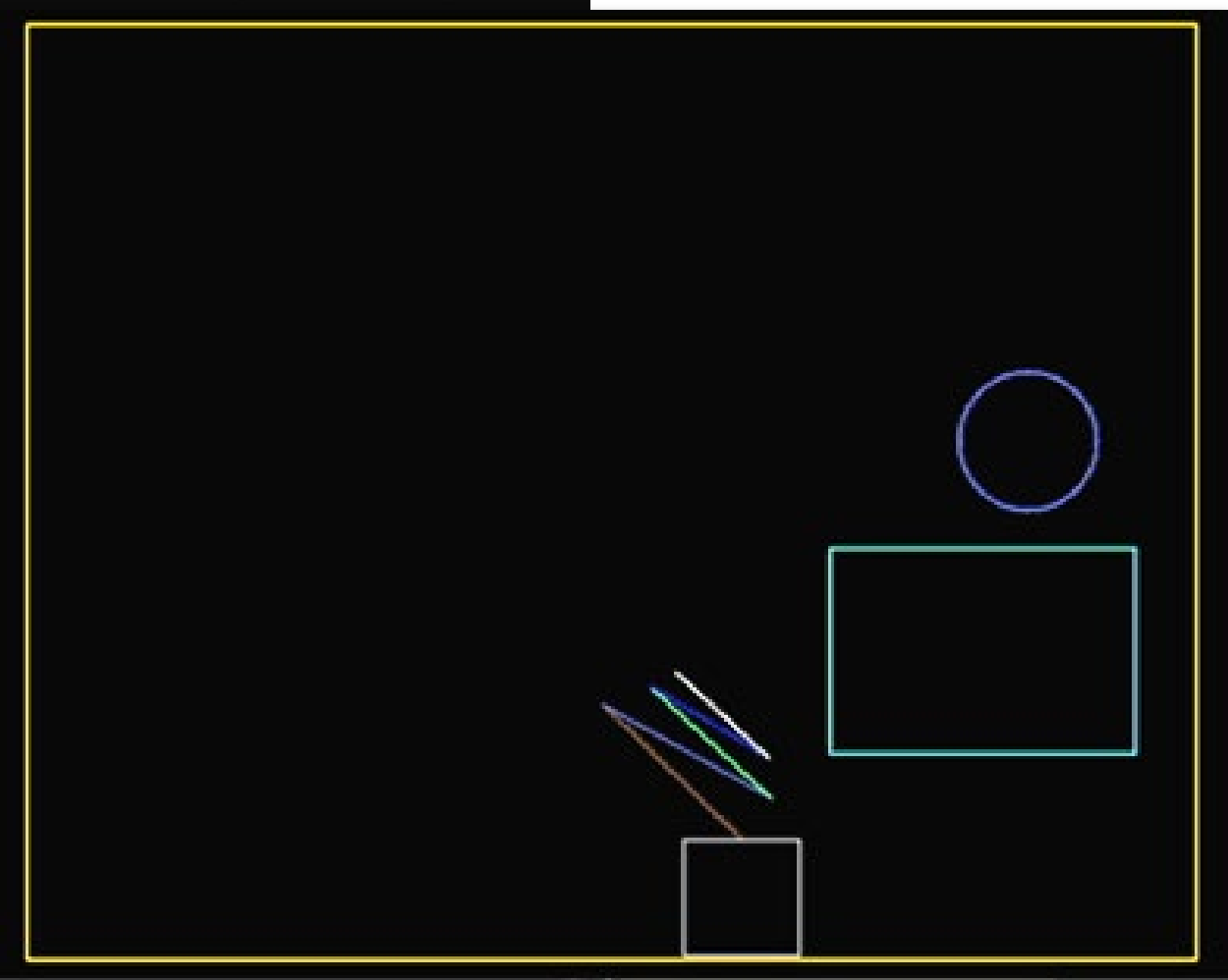









\section{Scaling}

II/ Robot Base, Tunnel, Magnet, and Pipe + Scaling

int border $=5$;

int pixelwidth $=$ windoww $-(2 *$ border $)$;

int pixelheight $=$ windowh $-(2 *$ border $)$;

int basewidth $=12$;

int baseheight $=12$

//Base width (inches)

//Base height (inches)

int tunnelwidth $=120$

int tunnelheight $=96$;

int tunnelwpixels $=0$;

int tunnelhpixels $=\theta$;

int scaleheight = pixelheight / tunnelheight;

int scalewidth $=$ pixelwidth $/$ tunnelwidth;

float scale = scaleheight;

if (scalewidth < scaleheight) \{

scale = scalewidth;

\}

int basewidthpixels = (int) (basewidth * scale);

int baseheightpixels = (int) (baseheight * scale);

int base xcenter;

1 float base_ycenter $=13$;

int base_ycenterpixels $=($ int $)$ (base_ycenter * scale)

tunnelhpixels $=$ (int) $($ tunnelheight $*$ scale $) ;$

tunnelwpixels = (int) (tunnelwidth * scale); $\gamma$ Window Border
// Tunnel width (Inches)

// Tunnel Height (Inches)

// Tunnel width (Pixels)

// Tunnel Height (Pixels)

// Scaling Tunnel Height

// Scaling Tunnel width
Tunnel width and height (Inches)

Setting variable to zero
$/ /$ Base Inches to Pixels

// Base- Inches off the Wa11

// Base- Inches off the Ground

/I Inches to Pixel:
//Base Inches to Pixels

Scaling \& converting inches to pixels

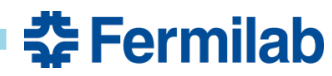




\section{Scaling}



Scaling \& converting inches to pixels Center of Base 


\section{Key Events}

while (SDL_Pollevent(\&event) $!=0$ ) \{

if (event.type $==$ SDL_QUIT) \{

quit $=1$;

if (event. type $==$ SDL_KEYDOWN) \{

if (event. key. keysym.sym == SDLK_q) \{

rotate $=$-rotationspeed;

Moves

if (event.key.keysym.sym == SDLK_a)

rotate $=$ rotationspeed

arm is

if (event. key. keysym. sym == SDLK w) rotate 2 = -rotationspeed;

if (event.key.keysym.sym $==$ SDLK_s) \{ rotate 2 = rotationspeed;

Stops

movement if (event.key.keysym.sym == SDLK_q) \{ rotate $=0.0$;

if (event. key. keysym. sym == SDLK_a) f rotate $=0.0$; \}.

if (event.key.keysym.sym == SDLK_w) \{ rotate $2=0.0$;

3.

if (event. key.keysym.sym == SDLK_s) \{ rotate $2=0.0$; 
int lengthpixels $=($ int $)($ length * scale); S C a le S link

LineLX $=(($ windoww) $-($ tunnelwpixels $)$ +tunnelwpixels - base_xcenterpixels - (basewidthpixels / 2)) + (basewidthpixels / 2); LineLY $=($ windoww $)-($ tunnelwpixels $)+$ tunnelhpixels - base_ycenterpixels - (baseheightpixels / 2);

$/ /$ Line One direction

LineA = LineA + rotate;

//Locations of Line One corners

Line RX $=$ LineLX $+(($ lengthpixels $/ 2.0) * \cos ($ LineA $*(p i / 180.0)))-(($ lengthpixels / 2.0) $* \sin ($ LineA $*(p i / 180.0)))$; LineRY $=$ LineLY $+(($ lengthpixels / 2.0) $* \sin ($ LineA $*(p i / 180.0)))+(($ lengthpixels / 2.0) $* \cos ($ LineA $*(p i / 180.0)))$;

//Draw Line One

SDL_SetRenderDrawColor (renderer, 220, 20, 60, 255);

SDL_RenderDrawLine(renderer, LineLX, LineLY, LineRX, LineRY);

//LINE TWO INFO

int length2 oixels $=($ int $)($ length $2 *$ scale $):$

//Line Two starting location

LineLX2 = LineRX;

LineLY2 = LineRY

//Line Two direction

if (rotate $==0.0)\{$ \}

LineA2 $=$ LineA2 + rotate 2

else \{ j

LineA2 = LineA2 + rotate;

//Locations of Line Two corners

LineRX2 $=$ LineLX2 $+(($ length2pixels $/ 2.0) * \cos ($ LineA2 $*(p i / 180.0)))-(($ length2pixels $/ 2.0) * \sin ($ LineA2 $*(p i / 180.0))) ;$ LineRY2 $=$ LineLY2 $+(($ length2pixels $/ 2.0) * \sin ($ LineA2 $*(p i / 180.0)))+(($ length2pixels / 2.0) $* \cos ($ LineA2 $*(p i / 180.0))) ;$

//Draw Line Two

SDL_SetRenderDrawColor (renderer, 90, 79, 207, 255);

SDL_RenderDrawLine(renderer, LineLX2, LineLY2, LineRX2, LineRY2);
-Enter distance between the center of the base and the wall (in inches):46 Enter number of arm links $(1-6): 5$

Enter length of Link One (in inches):28

Enter length of Link Two (in inches): 28

Enter length of Link Three (in inches) 24

Enter length of Link Four (in inches):20

:Enter length of Link Five (in inches):18 
if (linktotal $=2)\{$

int lengthpixels $=($ int $)($ length $*$ scale $)$;

//Centers Line One in the window

LineLX $=(($ windoww $)-($ tunnelwpixels $)+$ tunnelwpixels - base_xcenterpixels $-($ basewidthpixels / 2)) $+($ basewidthpixels / 2);

LinelY $=($ windoww $)-($ tunnelwpixels $)+$ tunnelhpixels - base_ycenterpixels - (baseheightpixels / 2);

//Line One direction

Line $A=$ LineA + rotate;

//Locations of Line One corners

LineRX $=$ LineLX $+(($ lengthpixels $/ 2.0) * \cos ($ LineA $*(p i / 180.0)))-(($ lengthpixels $/ 2.0) * \sin ($ LineA $*(p i / 180.0))) ;$

LineRY $=\operatorname{LineLY}+(($ lengthpixels $/ 2.0) * \sin ($ LineA $*(p i / 180.0)))+(($ lengthpixels / 2.0) $* \cos ($ LineA $*(p i / 180.0)))$;

//Draw Line One

SDL_SetRenderDrawColor (renderer, 220, 20, 60, 255);

SDL RenderDrawLine(renderer, LineLX, LineLY, LineRX, LineRY);

//LINE TWO INFO

int length2pixels $=($ int $)($ length $2 *$ scale $)$;

//Line Two starting location

LineLX2 = LineRX

LineLY2 = LineRY;

if (rotate directio

(rotate $==0.0)$

Line $A 2=$ Line $A 2+$ rotate 2

else \{

Sets line angle

LineA2 $=$ LineA2 + rotate

//Locations of Line Two corners

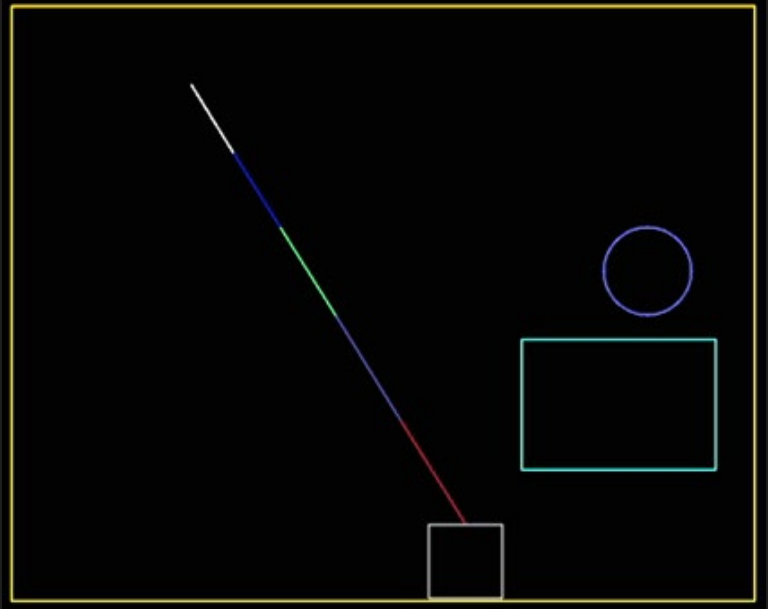

LineRX2 $=$ LineLX2 $+(($ length2pixels $/ 2.0) * \cos ($ LineA2 $*($ pi $/ 180 . \theta)))-(($ length2pixels $/ 2 . \theta) * \sin ($ LineA2 $*(p i / 180 . \theta)))$;

LineRY2 $=$ LineLY2 $+(($ length2pixels $/ 2.0) * \sin ($ LineA2 $*(p i / 180.0)))+(($ length2pixels / 2.0) $* \cos ($ LineA2 $*(p i / 180.0))) ;$

//Draw Line Two

SDL SetRenderDrawColor(renderer, 90, 79, 207, 255);

SDL_RenderDrawLine(renderer, LineLX2, LineLY2, LineRX2, LineRY2); 
if (linktotal $=2)\{$

int lengthpixels $=($ int $)($ length $*$ scale $)$;

//Centers Line One in the window

LineLX $=(($ windoww $)-($ tunnelwpixels $)+$ tunnelwpixels - base_xcenterpixels $-($ basewidthpixels / 2)) $+($ basewidthpixels / 2); LineLY $=($ windoww $)-($ tunnelwpixels $)+$ tunnelhpixels - base_ycenterpixels $-($ baseheightpixels / 2);

$/ /$ Line One direction

Line $A=$ Line A + rotate;

//Locations of Line One corners

LineRX $=$ LineLX $+(($ lengthpixels $/ 2.0) * \cos ($ LineA $*(p i / 180.0)))-(($ lengthpixels $/ 2.0) * \sin ($ LineA $*(p i / 180.0))) ;$ LineRY $=\operatorname{LineLY}+(($ lengthpixels $/ 2.0) * \sin ($ LineA $*(p i / 180.0)))+(($ lengthpixels $/ 2.0) * \cos ($ LineA $*(p i / 180.0)))$;

//Draw Line One

SDL_SetRenderDrawColor(renderer, 220, 20, 60, 255);

SDL_RenderDrawLine(renderer, LineLX, LineLY, LineRX, LineRY);

//LINE TWO INFO

int length2pixels $=($ int $)($ length2 $*$ scale $) ;$

//Line Two starting location

LineLX2 = LineRX

LineLY2 = LineRY

/ Line Two direction

if $($ rotate $=0.0)\{$ 了 LineA2 $=$ LineA2 + rotate 2

LineA2 $=$ LineA2 + rotate;

//Locations of Line Two corners

LineRX2 $=$ LineLX2 $+(($ length2pixels $/ 2.0) * \cos ($ LineA2 $*(p i / 180.0)))-(($ length2pixels $/ 2.0) * \sin ($ LineA2 $*(p i / 180.0))) ;$ LineRY2 $=$ LineLY2 $+(($ length2pixels $/ 2.0) * \sin ($ LineA2 $*(p i / 180.0)))+(($ length2pixels $/ 2.0) * \cos ($ LineA2 $*(p i / 180.0))) ;$

//Draw Line Two

SDL_SetRenderDrawColor(renderer, 90, 79, 207, 255);

SDL_RenderDrawLine(renderer, LineLX2, LineLY2, LineRX2, LineRY2); 
SDL_SetRenderDrawColor(renderer, 255, 225, 0, 255);

dstrect. $x=$ (windoww / 2) - (tunnelwpixels / 2);

dstrect.y $=($ windowh $/ 2$ ) - (tunnelhpixels / 2);

dstrect.w = tunnelwpixels;

dstrect.h = tunnelhpixels;

SDL_RenderDrawRect(renderer, \&dstrect);

\section{Rendering the} Tunnel 
dstrect. $\mathrm{x}=$ (windoww $/ 2$ ) - (tunnelwpixels $/ 2$ );

dstrect.y $=($ windowh $/ 2$ ) - (tunnelhpixels / 2);

dstrect.w = tunnelwpixels;

dstrect.h = tunnelhpixels;

SDL_RenderDrawRect(renderer, \&dstrect);

Rendering the

Tunnel 
SDL_SetRenderDrawColor(renderer, 255, 225, 0, 255);

dstrect. $\mathrm{x}=($ windoww $/ 2)-($ tunnelwpixels / 2);

dstrect.y $=($ windowh $/ 2)-($ tunnelhpixels / 2);

\section{Set Location}

\section{Rendering the Tunnel}


Render Tunnel

SDL_SetRenderDrawColor(renderer, 255, 225, 0, 255);

dstrect. $x=$ (windoww / 2) - (tunnelwpixels / 2);

dstrect.y $=$ (windowh $/ 2$ ) - (tunnelhpixels / 2);

dstrect.w = tunnelwpixels;

dstrect.h = tunnelhpixels;

SDL_RenderDrawRect(renderer, \&dstrect);

\section{Draw to Screen}

\section{Rendering the Tunnel}




\section{NX CAD}

\section{Robot Design}




\section{Long Reach Robotic Arm}

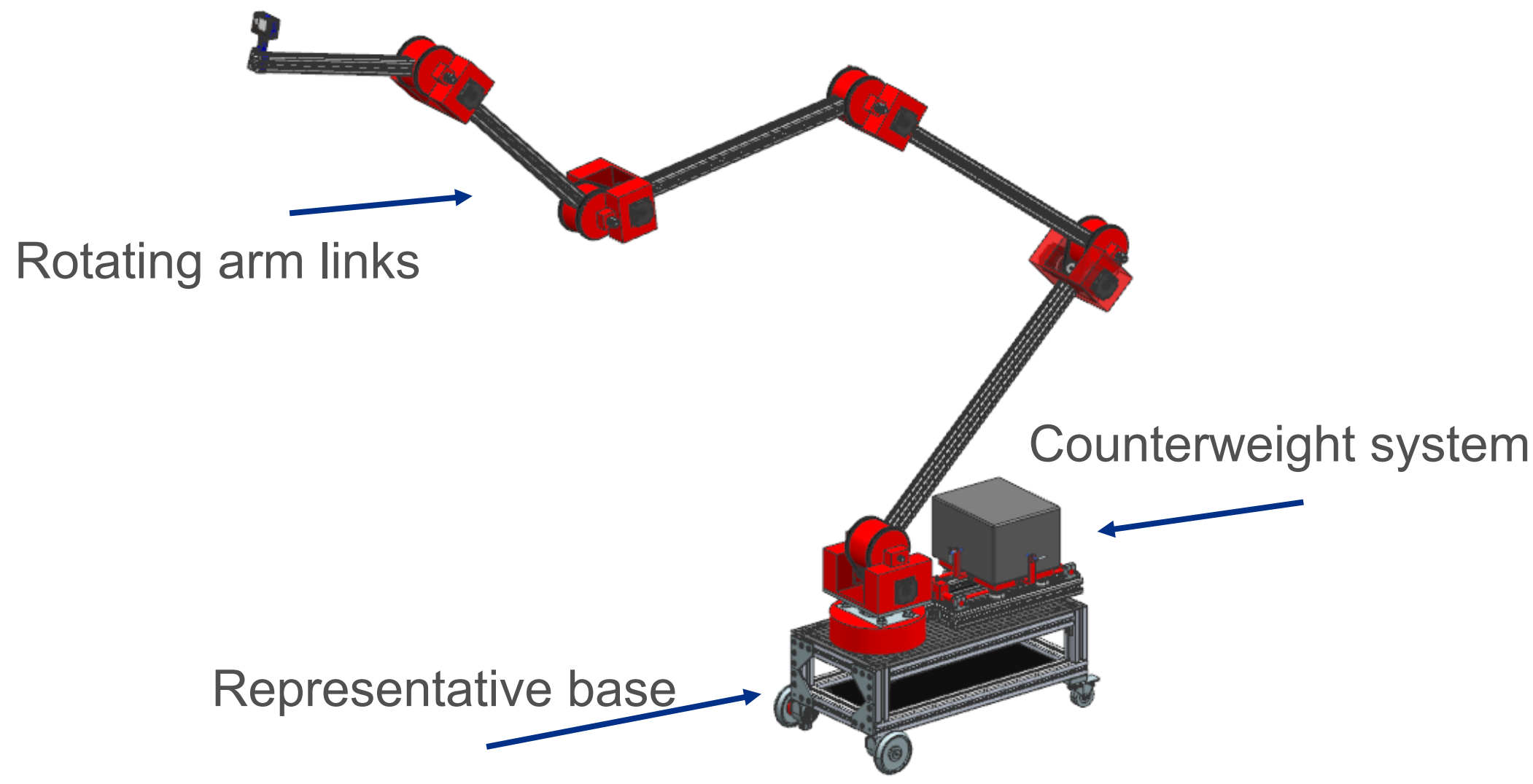




\section{Robot Arm Assembly}

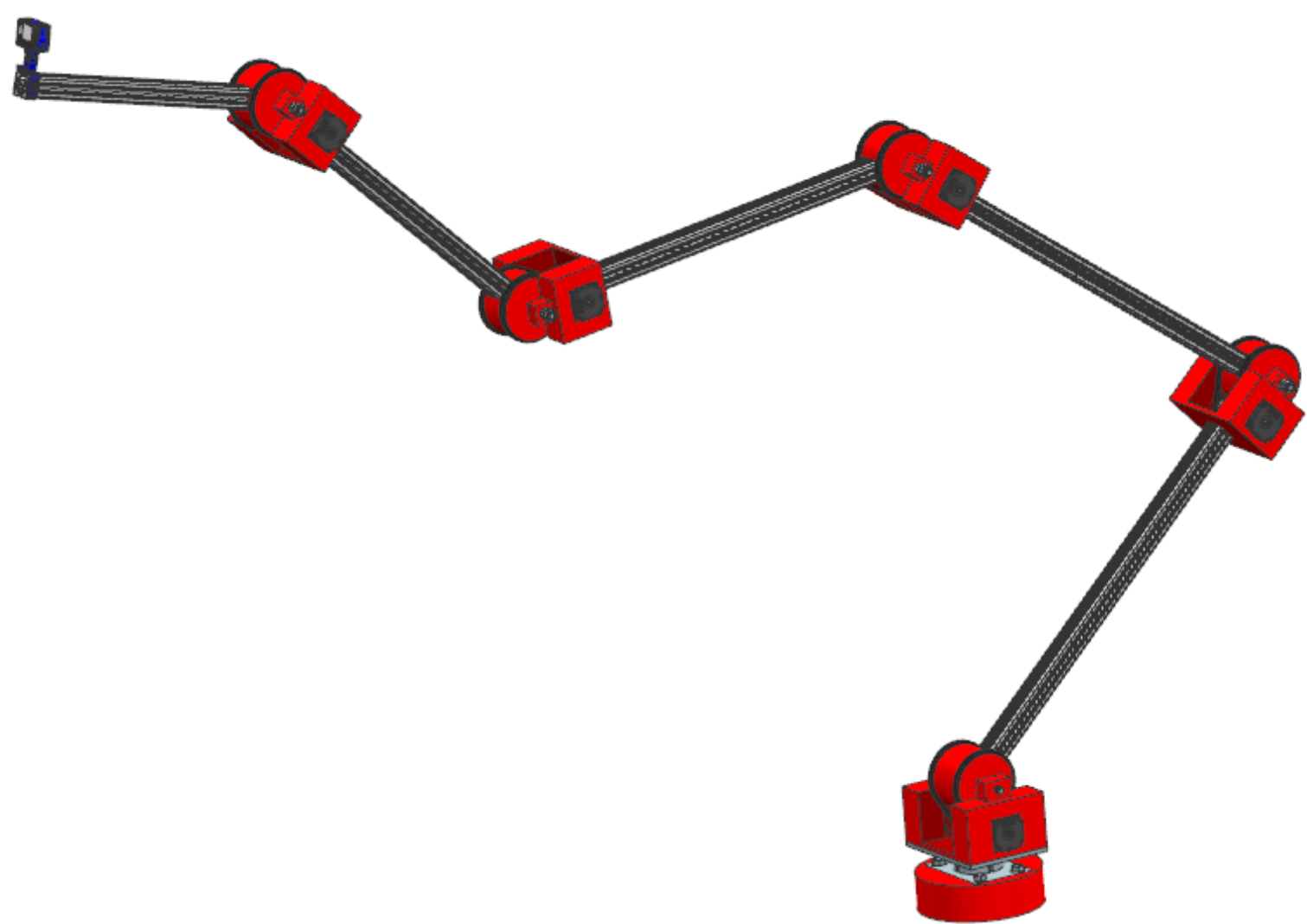




\section{Robot Arm Links}

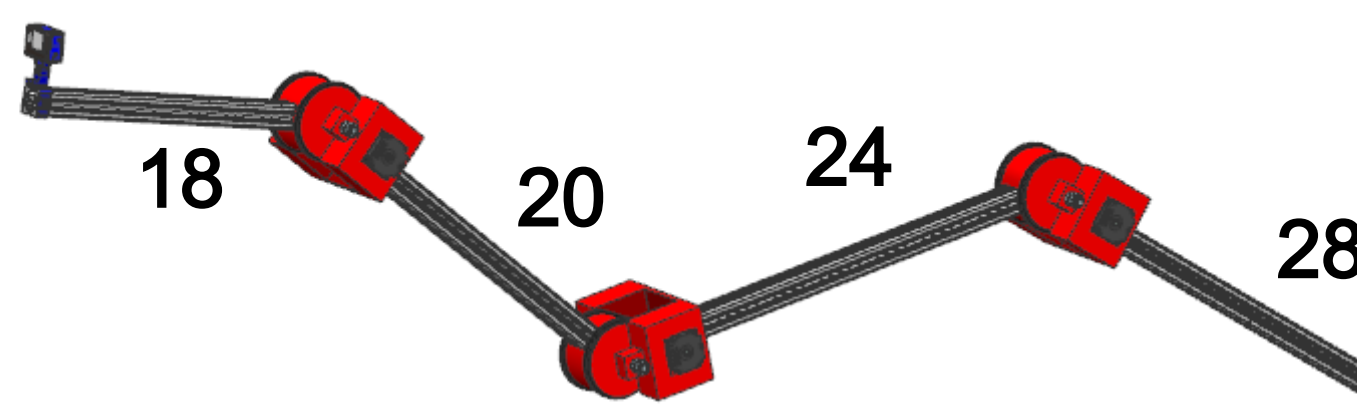

Enter number of arm links (1-6):5

Enter length of Link One (in inches):28

Enter length of Link Two (in inches):28

Enter length of Link Three (in inches) 24

Enter length of Link Four (in inches):20

Enter length of Link Five (in inches):18 


\section{Joint Assembly - Closer View}

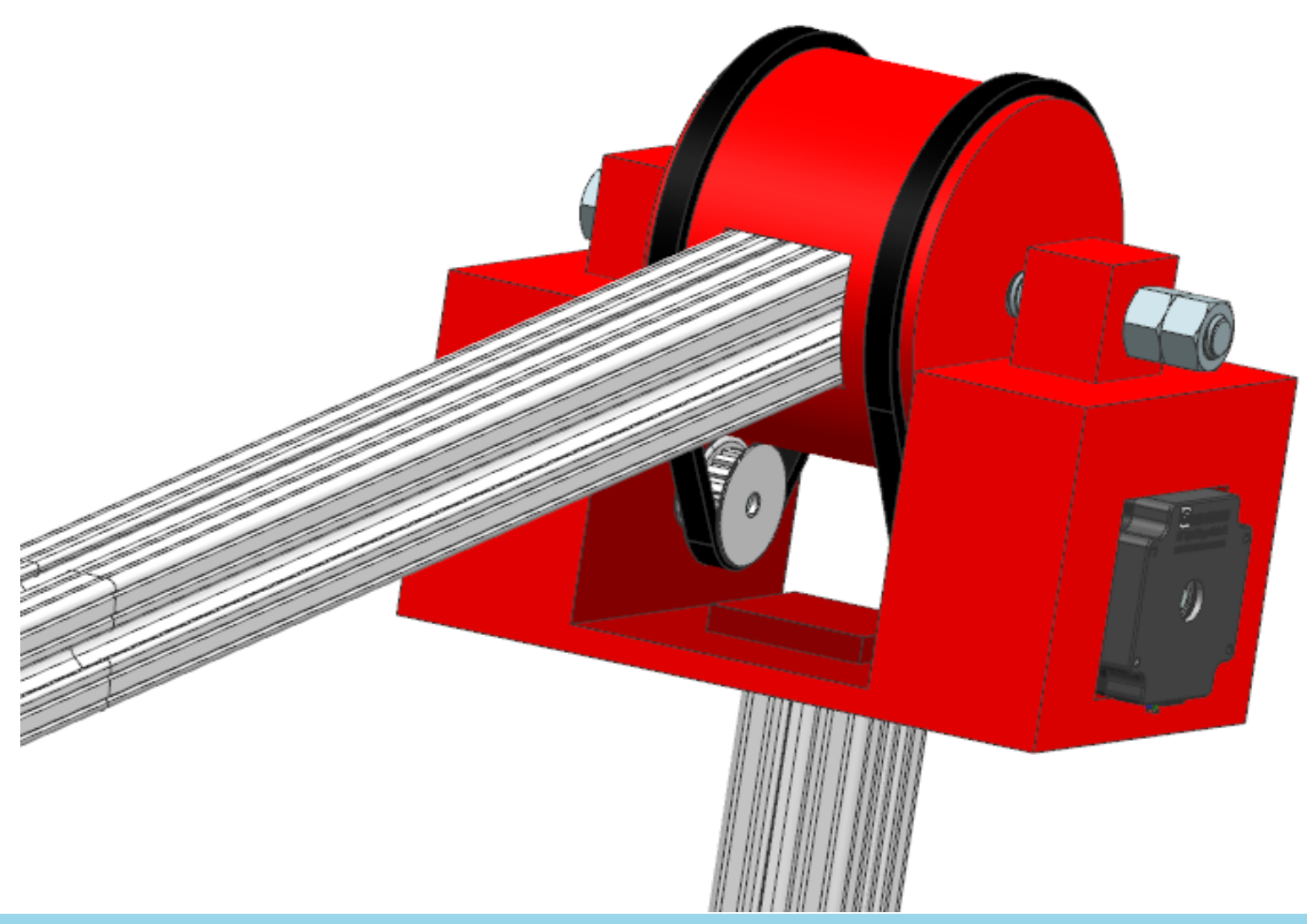




\section{Joint Assembly - How it Works}

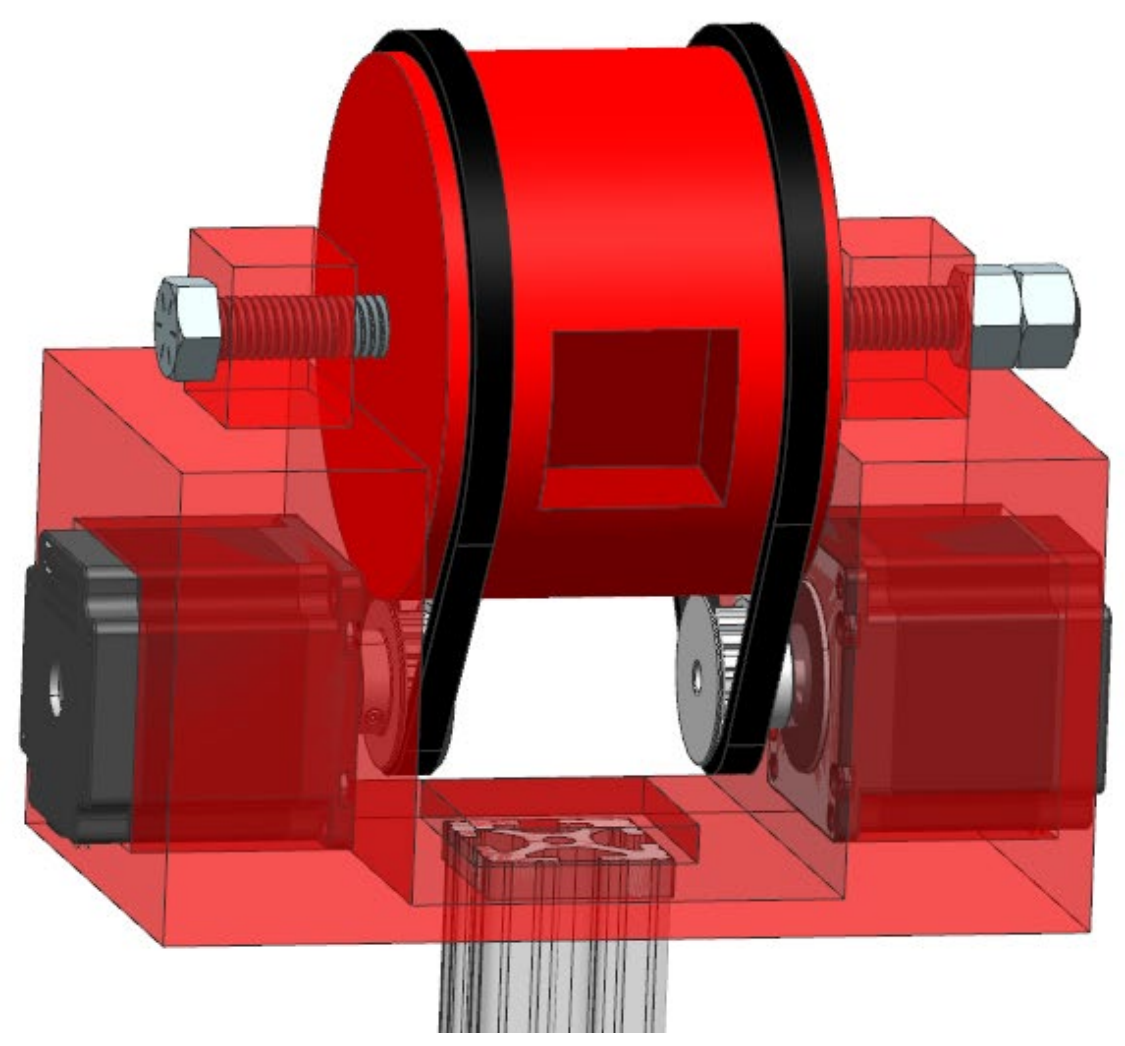




\section{Joint Assembly - How it Works}

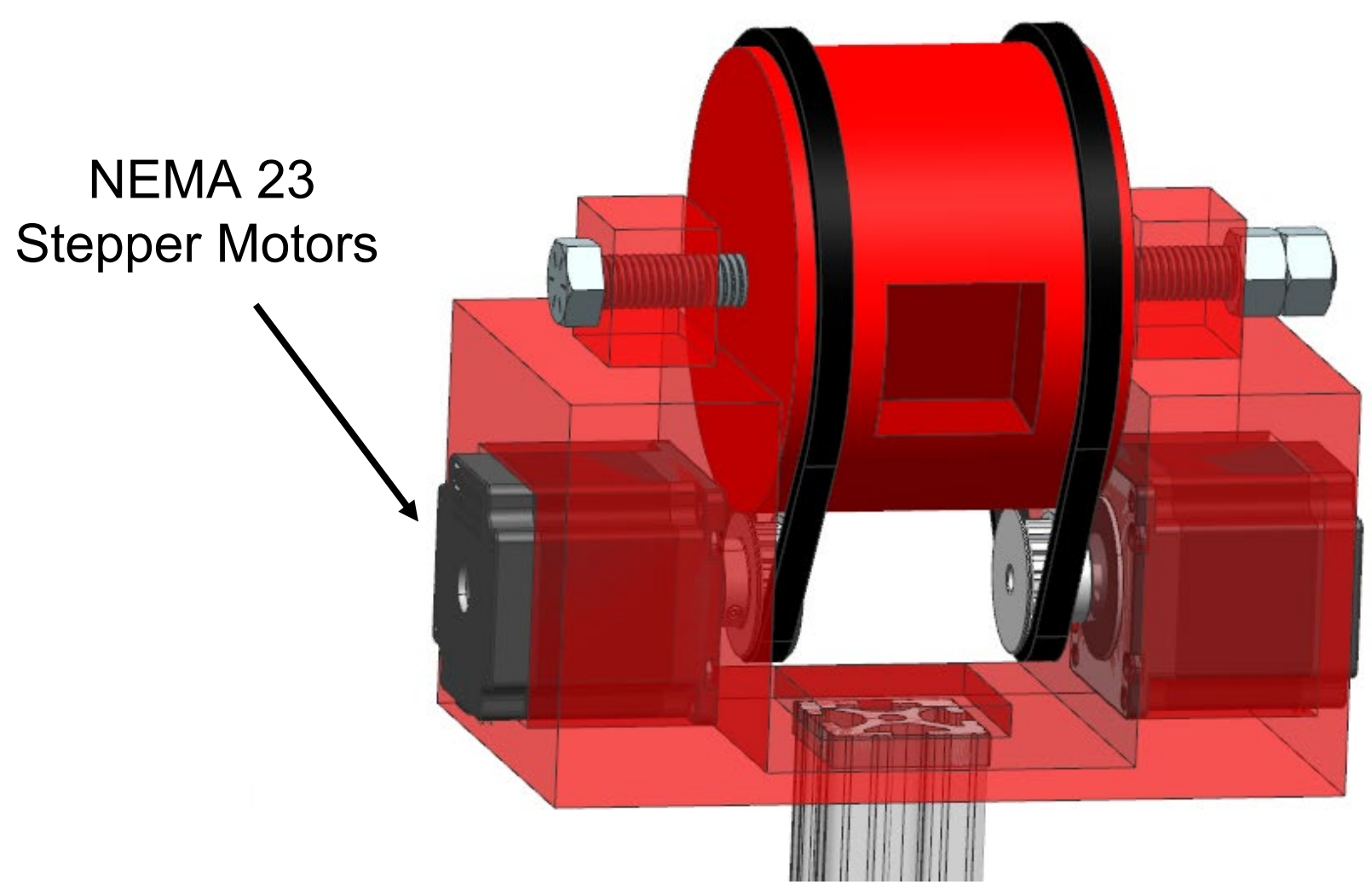




\section{Joint Assembly - How it Works}

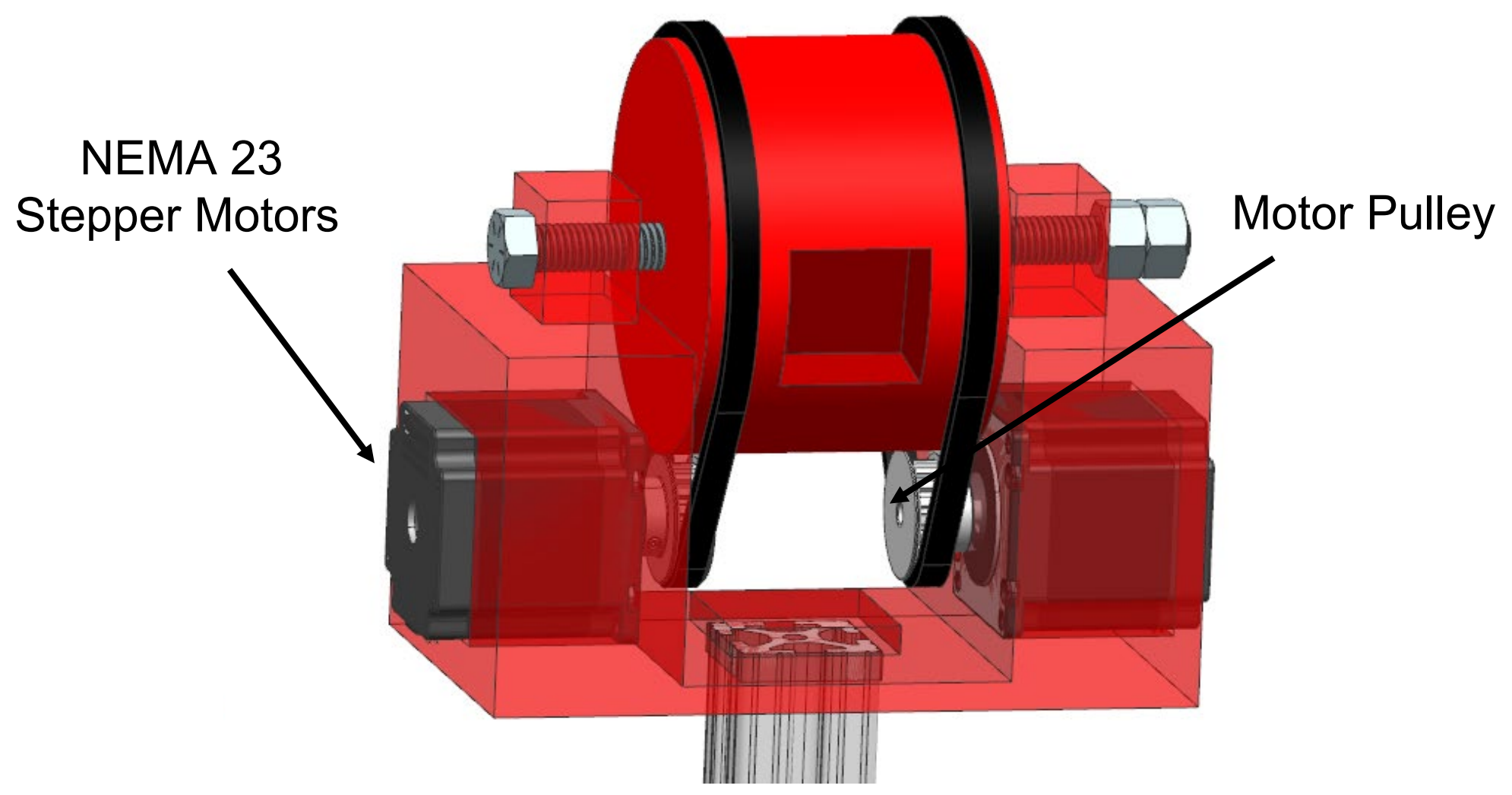




\section{Joint Assembly - How it Works}

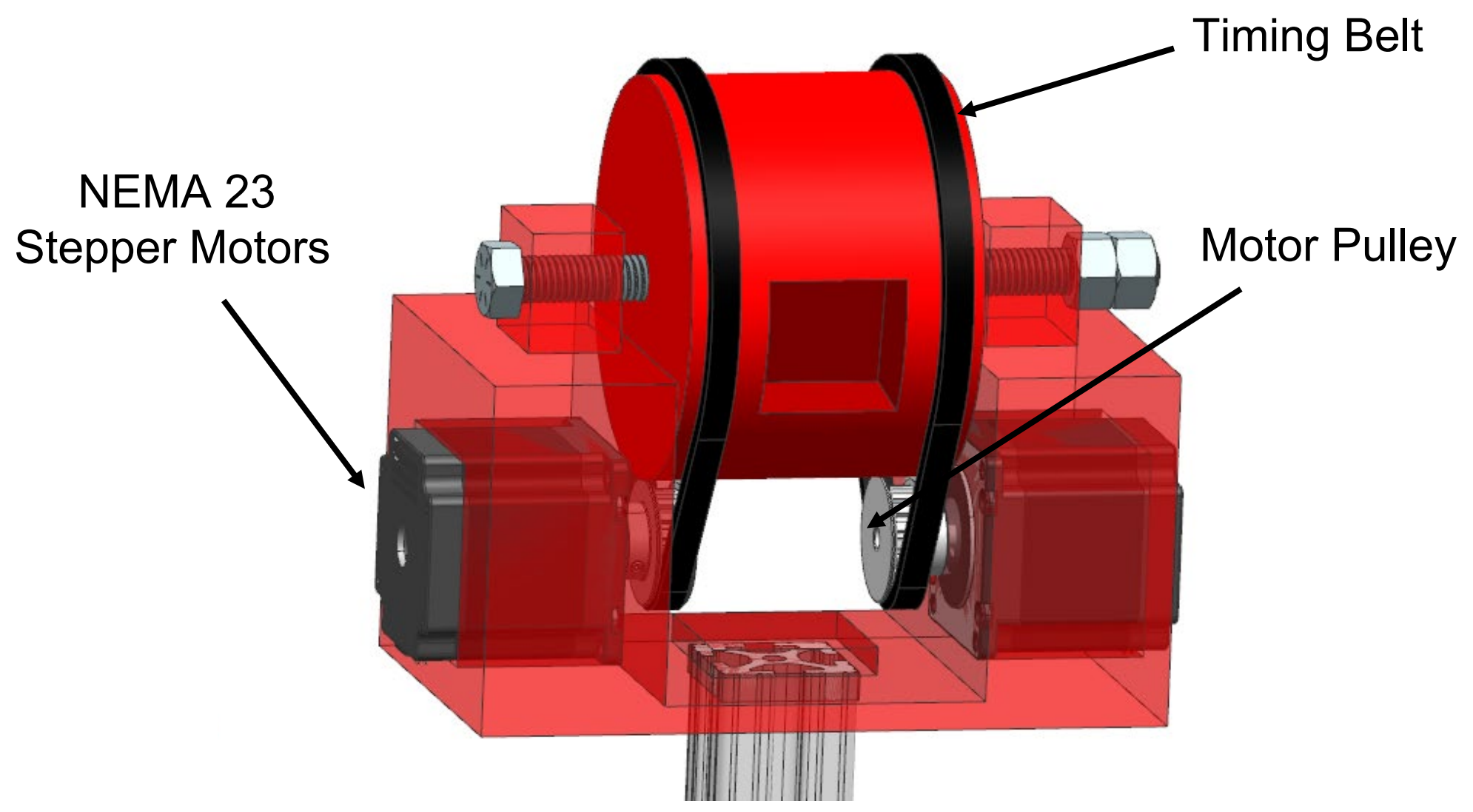




\section{Joint Assembly - How it Works}

7" - length hex head threaded bolt

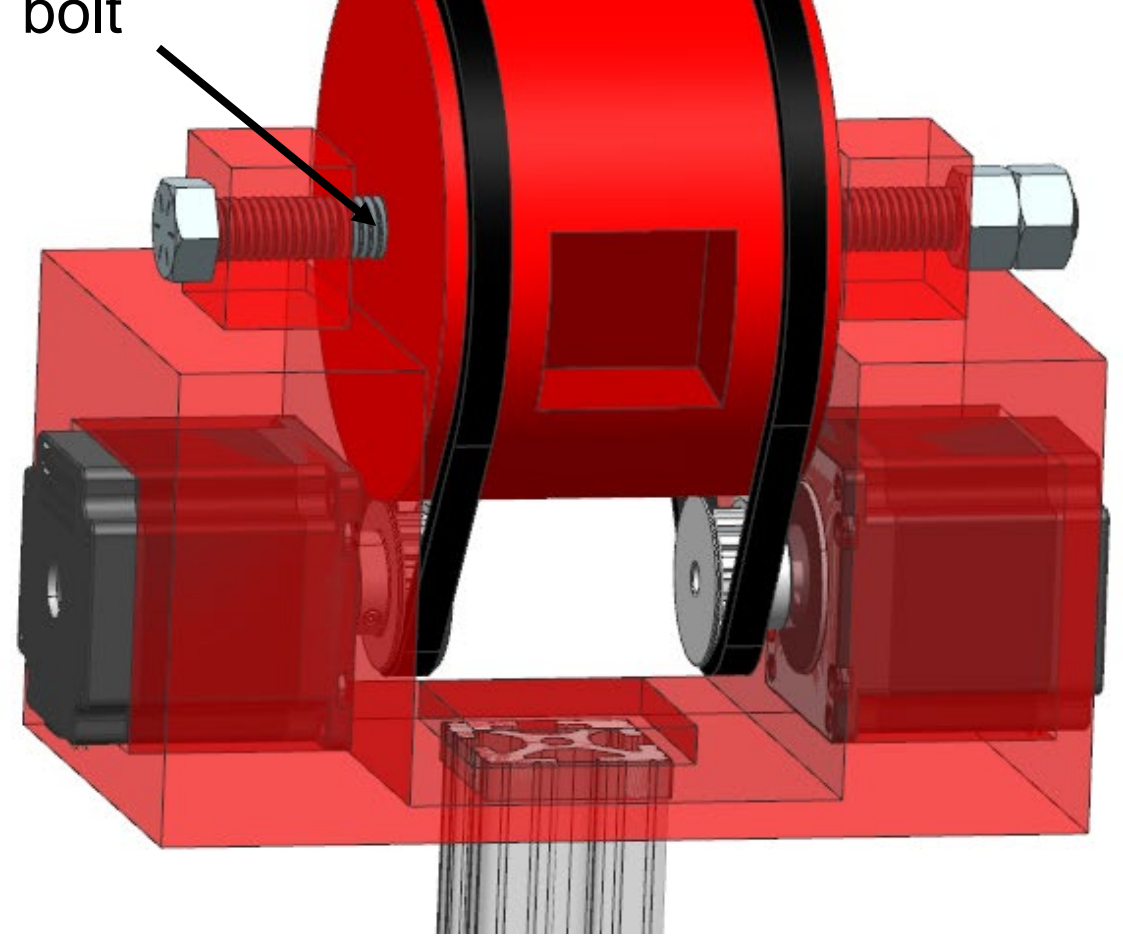




\section{Joint Assembly - How it Works}

7" - length hex head threaded bolt

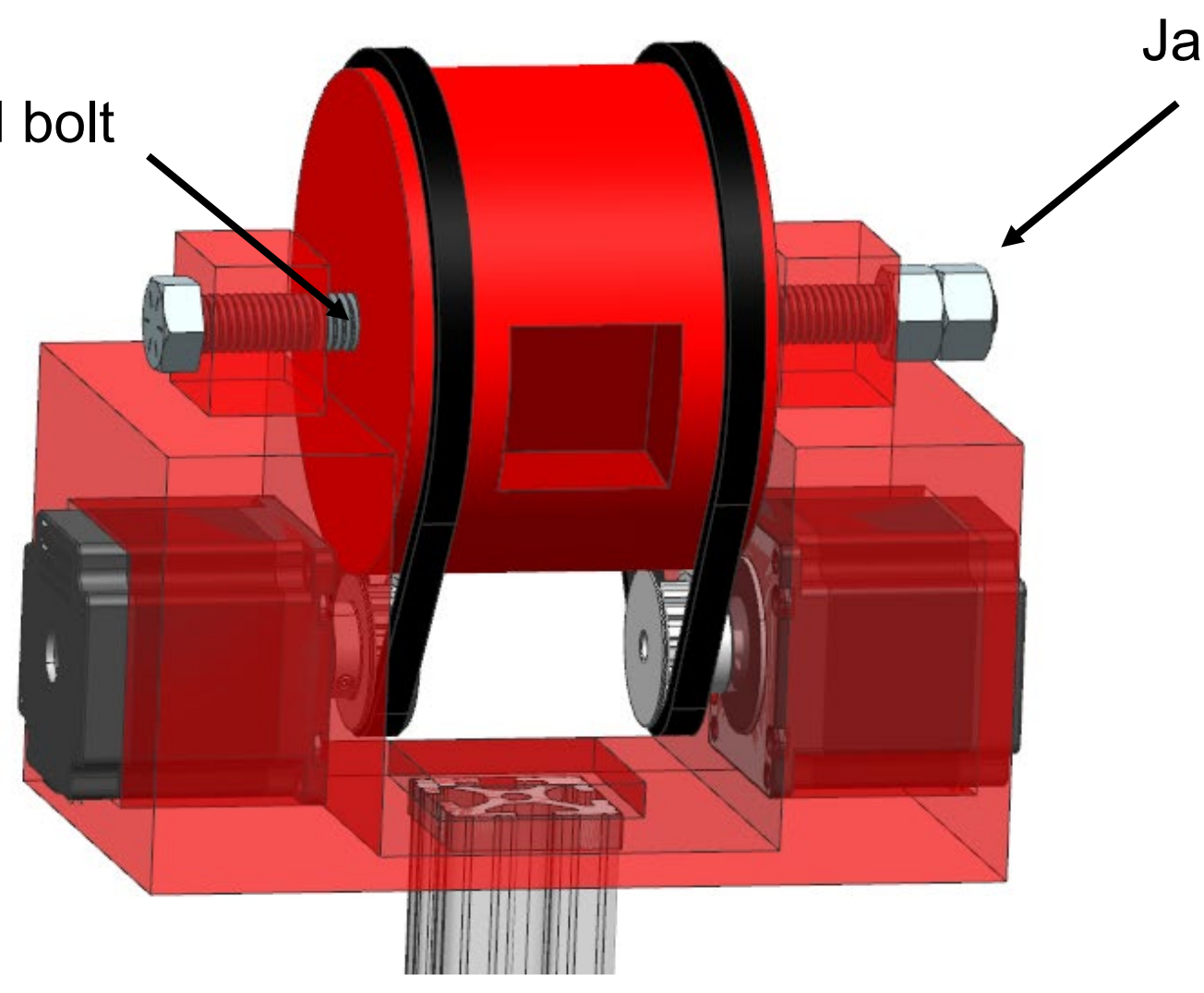




\section{GoPro Camera Mount}

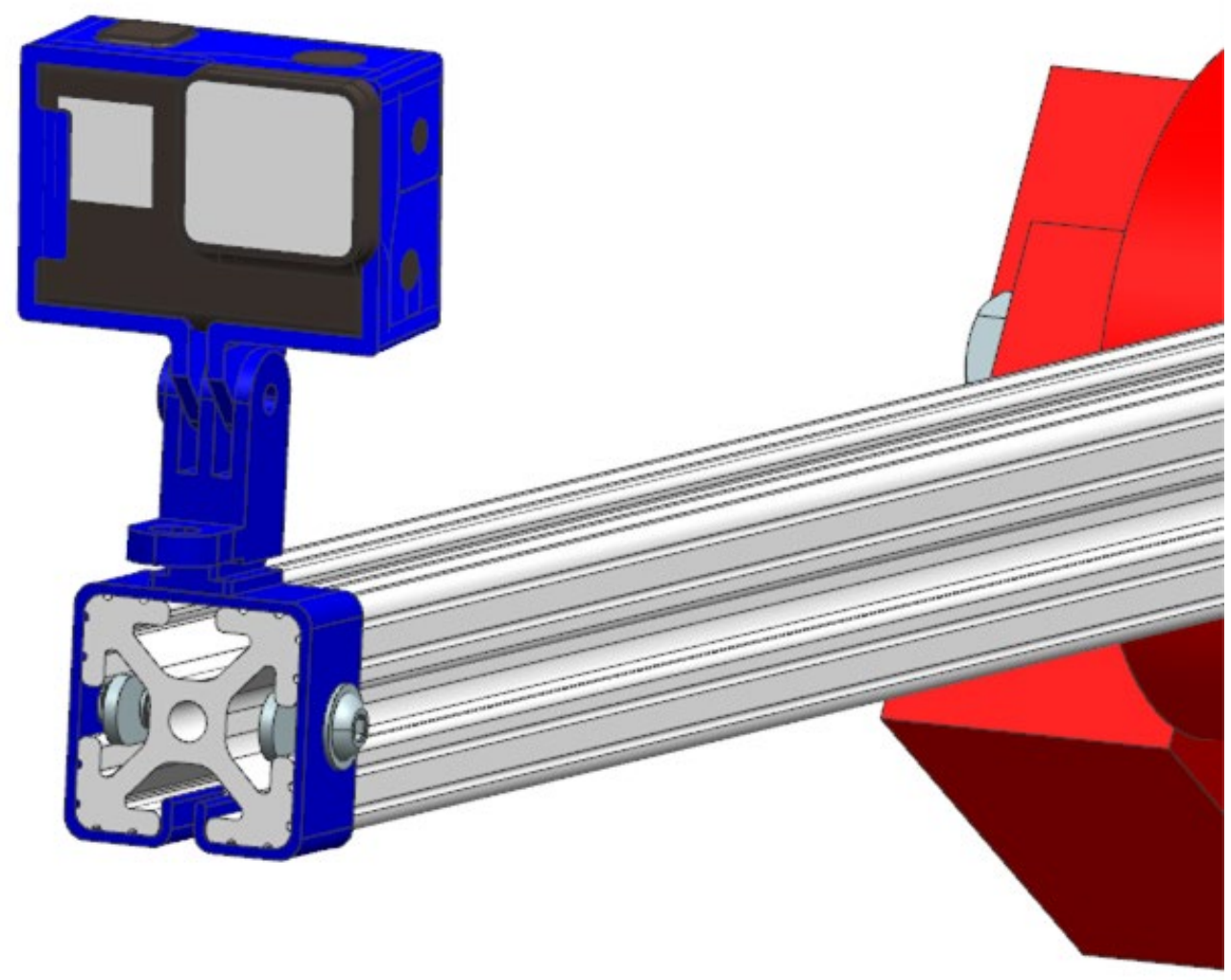




\section{The Counterweight Design}




\section{Counterweight Full Assembly}

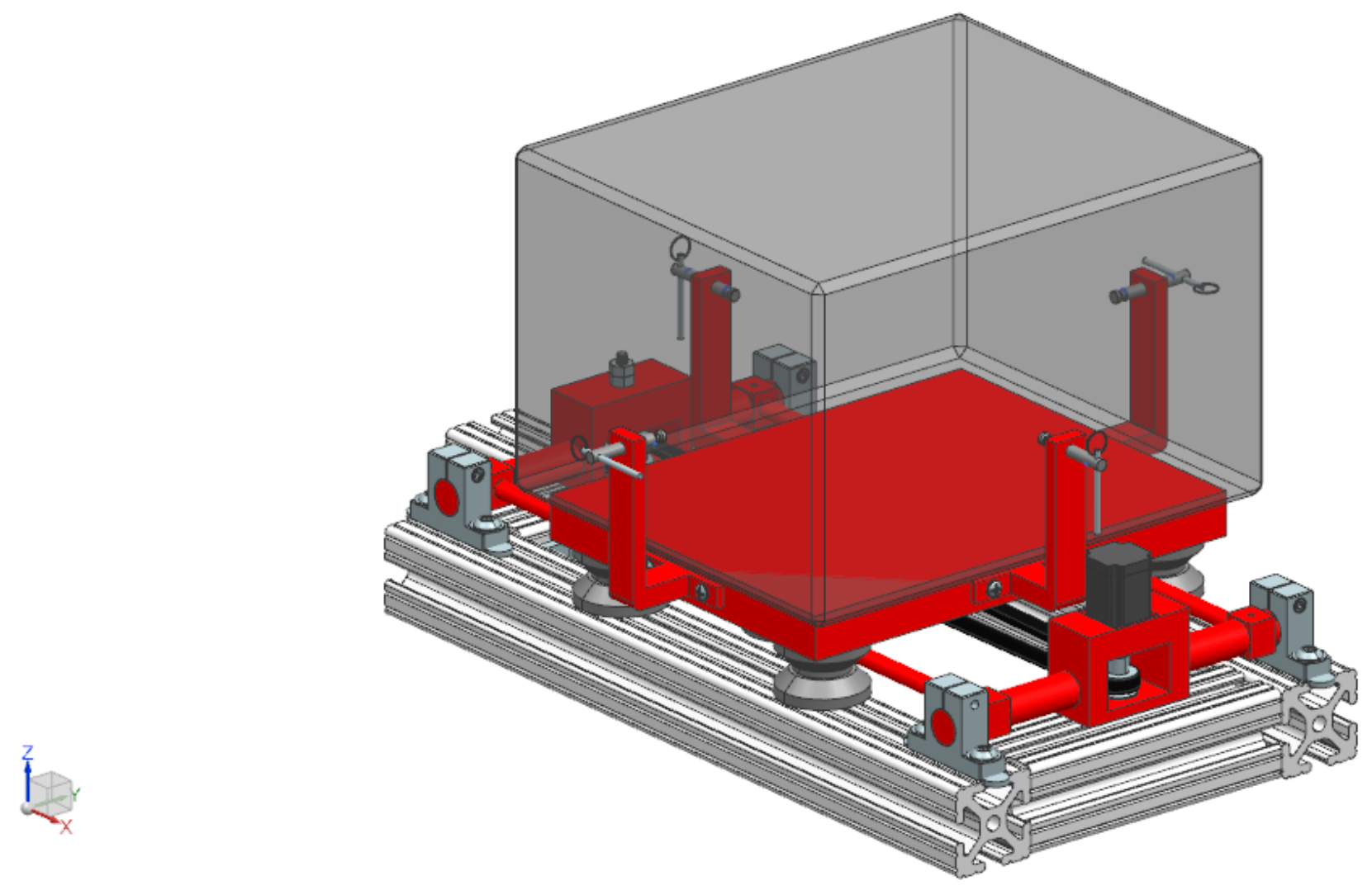




\section{Counterweight Iteration}

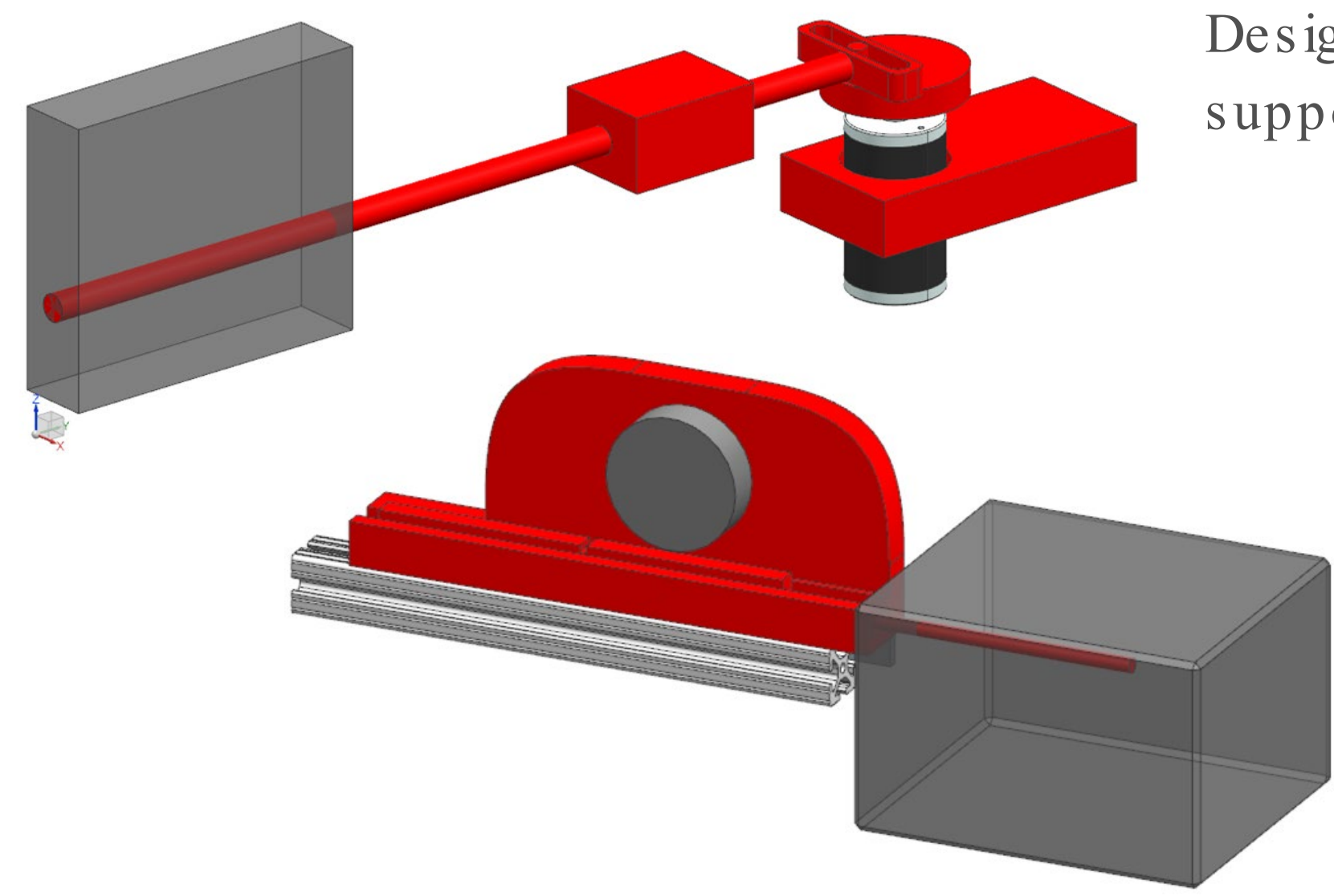

Design 2: limited horizontal movement 


\section{Counterweight Assembly}



110 lbs steel counterweight 


\section{Counterweight Assembly}

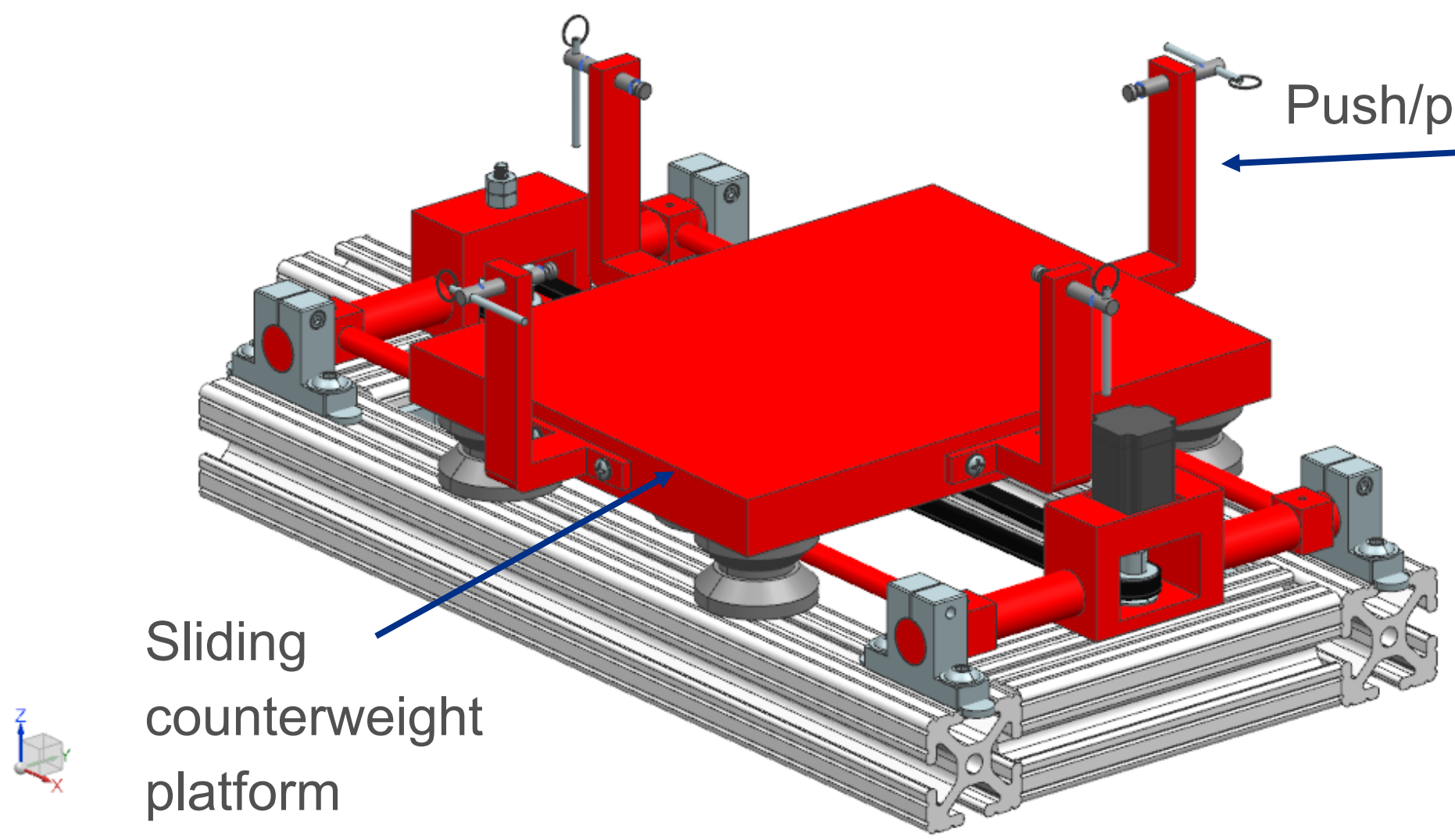




\section{Counterweight Assembly}

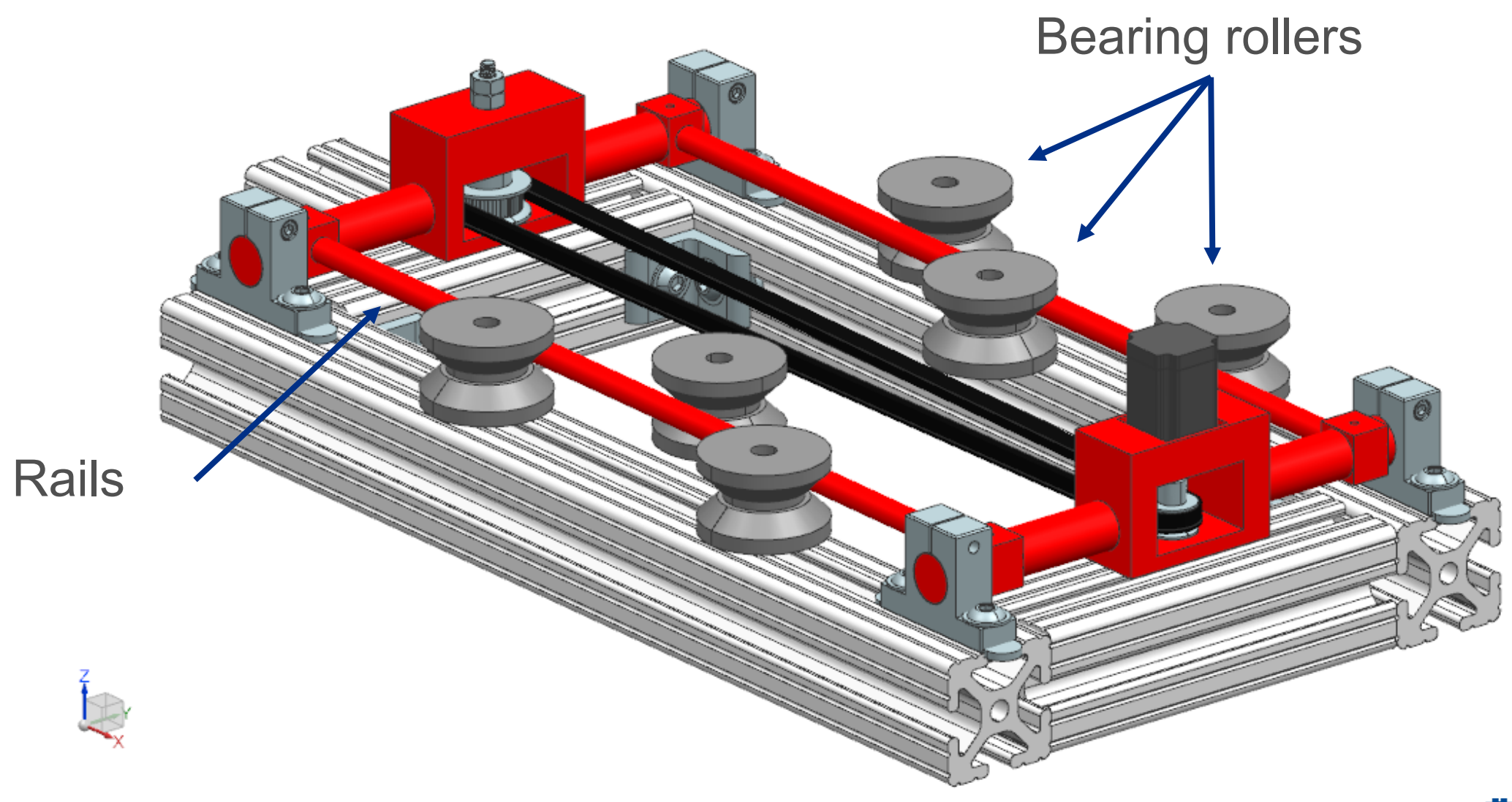




\section{Counterweight Assembly}

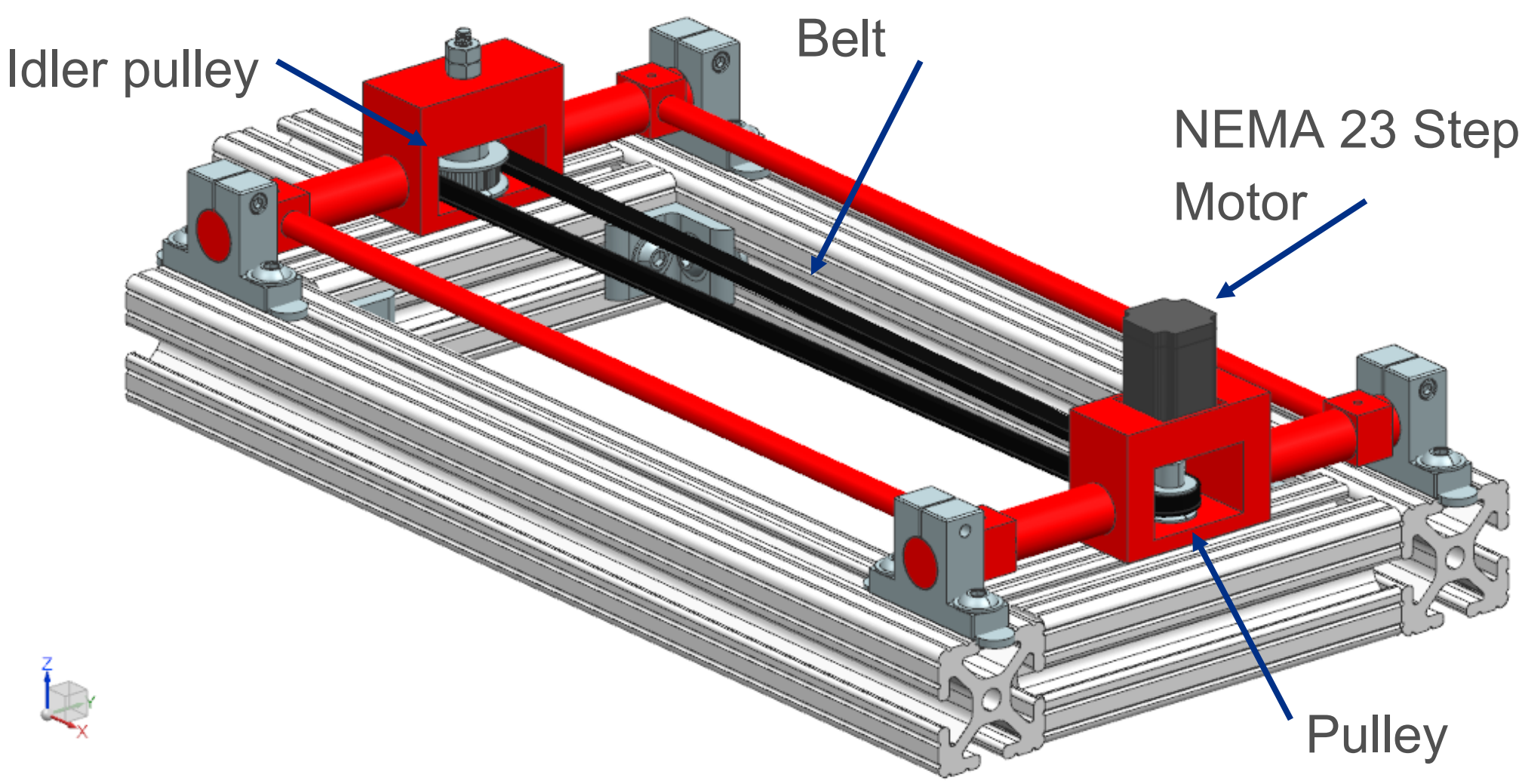




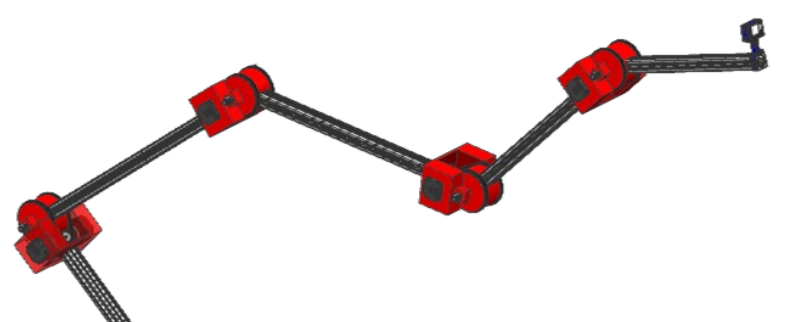

Thank you for attending our presentation!

$$
\text { Any questions? }
$$

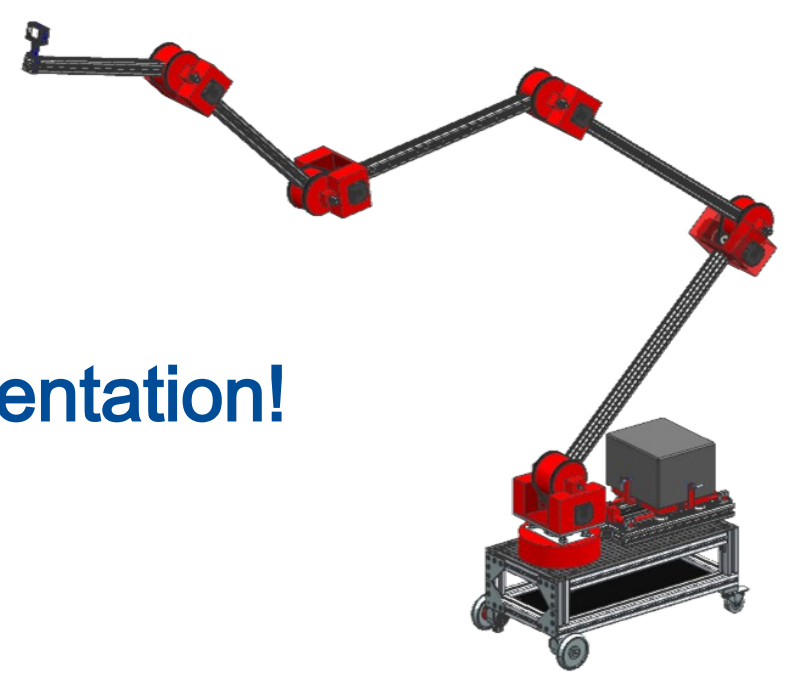

